\title{
Evaluation of drought tolerance from a wheat recombination inbred line population at the early seedling growth stage
}

\author{
Hong Zhang ${ }^{1,2}$ and Honggang Wang ${ }^{1 *}$ \\ ${ }^{1}$ State Key Laboratory of Crop Biology, Shandong Key Laboratory of Crop Biology, Taian Subcenter of National Wheat \\ Improvement Center, College of Agronomy, Shandong Agricultural University, Taian 271018, China. \\ ${ }^{2}$ Department of Agronomy, Dezhou University, Dezhou 253023, China.
}

Accepted 16 October, 2012

\begin{abstract}
The drought tolerance of wheat seedlings from a recombination inbred line (RIL) population derived from crosses between Weimai 8 and Luohan2 and its parents was evaluated according to the drought tolerance indices of 11 early seedling traits and using principal component analysis and K-means clustering methods to select materials with good germplasm. Results indicated that drought could promote the increase of coleoptile length (CL) and inhibit seedling height (SH), longest root length (RL), seedling fresh weight (SFW), shoots fresh weight (STFW), root fresh weight (RFW), seedling dry weight (SDW), shoots dry weight (STDW), root dry weight (RDW), root-to-shoot fresh weight ratio (RSFWR), and root-to-shoot dry weight ratio (RSDWR). Two hundred forty-five lines from a RIL population and two parents were divided into three clusters. The parent Weimai 8 and 101 lines were attributed to droughtsensitive types; the parent Luohan 2 and 102 lines mediated drought-resistant types, and 42 lines were highly drought-resistant types. Whether or not a relatively strong root system could be formed was the most important condition in evaluating the drought tolerance of wheat at the seedling stage.
\end{abstract}

Key words: Recombination inbred line, evaluation of drought tolerance, seedling growth stage, principal component analysis and cluster analysis.

\section{INTRODUCTION}

Seed germination and early seedling growth are considered to be the most critical stages for wheat establishment, especially under stress (Blum, 1996). The improvement of seedling drought tolerance of wheat variety can overcome the influence of water condition in soil, insure the basis seedling number and the establishment of photosynthesis population, and make solid base for obtaining high and stable yield. So the drought tolerance during seed germination and early seedling growth is stress tolerance trait that should not be neglected. Conventional evaluation method for drought tolerance was the yield evaluation under field drought condition, but this method needed a full season

\footnotetext{
*Corresponding author. E-mail: hgwang@sdau.edu.cn.
}

experiment with great workload and low efficiency, in addition, it did not adapt to evaluation of a mass of materials. Techniques of screening for drought tolerance within large number of cultivars should be easy, rapid and inexpensive. High molecular mass polyethylene glycol (PEG) has been widely used to mimic osmotic stress in culture solutions now (Almansouri et al., 2001), because this approach avoids much of the environmental noise associated with field experiments and induces a plant response similar to that induced by natural drought, for example, causing a depression in both seed germination and growth of the root and shoots (Dhanda et al., 2004; Mujtaba et al., 2005).

The objectives of this study were to evaluate the genetic variability of seedling drought tolerance among 245 lines of a recombination inbred line (RIL) population subjected to simulate drought conditions and to 
determine whether or not laboratory agronomy tests are useful criteria for screening of drought tolerance

\section{MATERIALS AND METHODS}

\section{Experimental material}

A F8:9 recombinant inbred line (RIL) populations derived from crosses between two Chinese common wheat varieties, Weimai 8 and Luohan 2, comprising 245 lines, was used in this present study. Weimai 8 is a drought sensitive variety, and it was released by Weifang Municipal Academy of Agricultural Sciences, Shandong, China in 2003; Luohan 2 is a drought tolerance variety, and it was released by Crop Research Institute, Luoyang Municipal Academy of Agricultural Sciences, Henan, China in 2001.

\section{Drought stress tests}

Drought stress tests were laid out according to the completely randomized design with three repeats. The osmotic potentials utilized were 0 (control) and $-0.1 \mathrm{MPa}$. Specific methods were as follows: One hundred seeds per each of $245 \mathrm{RIL}$ and their parents were packed in gauze, dipped into $3 \% \mathrm{H}_{2} \mathrm{O}_{2}$ for 10 min to sterilize them, washed 2 to 3 times using pure water, and soaked seeds to hasten germination for one day $(24 \mathrm{~h})$ at $25^{\circ} \mathrm{C}$. Then, for each material, sixty germination seeds were picked out and separately placed in six beakers $(6 \mathrm{~cm}$ wide) spread over two layers of filter paper, with ten each beaker. Three beakers were added $5 \mathrm{ml}$ of $10 \%(\Psi=-0.1 \mathrm{MPa})$ polyethylene glycol solution as treatment, and other three were added pure water as control. Calculation of osmotic potential used the equation of Michel and Kaufmann (1973).

$\Psi=[\mathrm{PEG}]^{2}[0.13 \mathrm{~T}-13.7]$

$\Psi=$ osmotic potential in MPa; [PEG] = concentration of polyethylene glycol (gram per gram of water); $\mathrm{T}=$ Temperature $\left({ }^{\circ} \mathrm{C}\right)$

All the beakers were placed in plastic boxes, covered with a thin plastic film, and cultured at $25^{\circ} \mathrm{C}$ in the dark for three days. On the fourth day, $5 \mathrm{ml}$ of water were added to every beaker with concurrent illumination $\left(50 \mu \mathrm{mol} \cdot \mathrm{m}^{-2} \cdot \mathrm{s}^{-1}\right)$ of $16 \mathrm{~h}$ every day. On the eighth day, five plants per beaker were randomly picked out to measure coleoptile length $(\mathrm{CL})$, seedling height $(\mathrm{SH})$, longest root length $(R L)$, seedling fresh weight (SFW), shoots fresh weight (STFW), and root fresh weight (RFW). The seedlings were placed in an oven for $20 \mathrm{~min}$ at $100^{\circ} \mathrm{C}$, and then dried to constant weight at $80^{\circ} \mathrm{C}$. The following parameters were subsequently measured: seedling dry weight (SDW), shoots dry weight (STDW), and root dry weight (RDW). Then, the root-to-shoot fresh weight ratio (RSFWR) and the root-to-shoot dry weight ratio (RSDWR) were calculated.

\section{Data analysis}

SAS (SAS, University of North Carolina, USA) was used in principal component analysis. The mean comparison of traits, Kmeans cluster, and correlation analysis were conducted using SPSS13.0 (SPSS, Chicago, USA). The drought tolerance index and subordinate function values of traits were calculated using the following formula:

$X=Y t / Y c \times 100 \%$ (Bouslama and Schapaugh 1984)]

$\mu(x)=(X-X \min ) /(X \max -X \min )($ He et al., 2008) where $\mathrm{X}$ is the drought tolerance index of trait, $\mathrm{Yt}$ is the trait value under stress, $Y_{c}$ is the trait value under normal water condition, $\mu(x)$ is the subordinate function value of some trait, $\mathrm{Xmin}$ is the minimal drought tolerance index value of some traits in all lines, and Xmax is the maximal drought tolerance index value of some traits in all lines. measurement value of drought tolerance (DV) for each line and parents was calculated by the subordinate function value of each trait multiplied by the eigenvector of corresponding trait of the first principal component and then added.

\section{RESULTS}

\section{Effects of drought on wheat seedling traits}

The results of the Paired-Samples T-test for 11 seedling traits of parents and RIL population indicated that the responses of two parents under osmotic stress conditions were different (Table 1). Under osmotic stress, the coleoptile length (CL) of two parents was increased, while SH, STFW, STDW, RL, SFW, SDW, RFW, and RDW were decreased. However, the increase of $\mathrm{CL}$ in Luohan 2 was greater than those of Weimai 8; for SH, STFW, STDW, the extent of decrease of the two parents was consistent. For RL, SFW, SDW, RFW, and RDW, the extent of decrease of Weimai 8 was greater than those of Luohan 2; for RSFWR, and RSDWR, Luohan 2 increased while Weimai 8 decreased. These findings indicate that the cultivar with drought tolerance has stronger rooting abilities to promote the absorption of water and decrease the effects of drought on growth.

For the RIL population, other traits decreased under osmotic stress conditions, except for CL which increased, indicating that osmotic stress could inhibit seedling growth but boost the elongation of the coleoptile to some extent. Under the two water conditions, the maximal value of all the traits for RIL population was larger than the parent with high trait value, but the minimal value was lower than the parents with small trait values.

\section{Principal component analysis}

The subordinate function value was calculated based on the drought tolerance index of each seedling trait of the RIL population and its parents. Each principal component eigenvector that amounts to weighting coefficient of each trait value and contribution rate was then calculated using principal component analysis of the subordinate function value with SAS software (Table 2). Different traits were plotted into different principal components based on the absolute value of each eigenvector. The greatest absolute value of the same trait lay in each factor that served as the principal component to which this trait belonged. Table 2 shows that the contribution rate of the first principal component was $40.8 \%$, which was decided mainly by the three variables, SFW, SDW, and RFW. These traits were all concerned with biological yield of the seedling, so the first principal component may be called 
Table 1. Mean comparison of seedling traits under two water conditions.

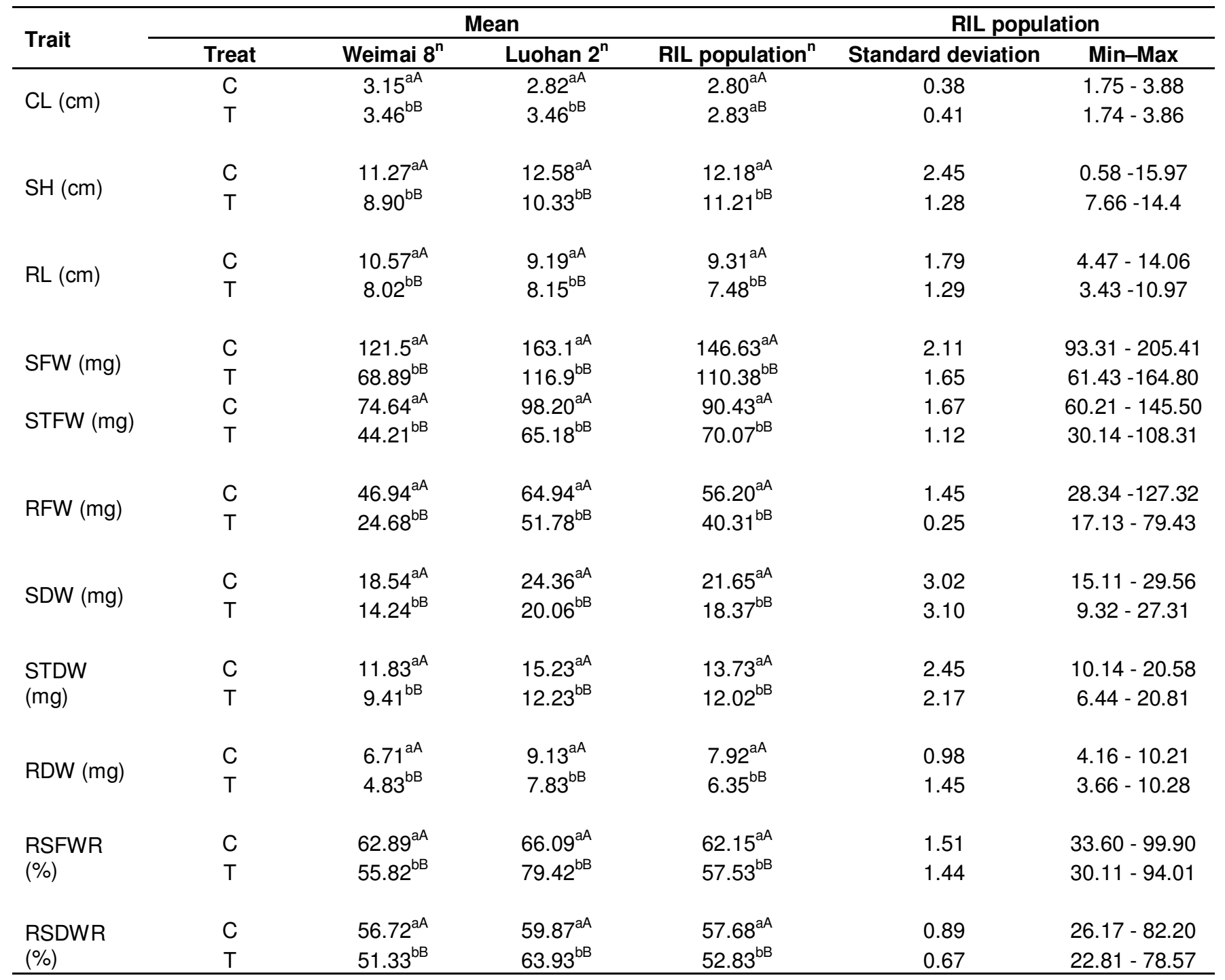

$\mathrm{C}$ and $\mathrm{T}$ present Control (normal water condition) and treatment (osmotic stress) respectively. CL, Coleoptile length, $\mathrm{SH}$, seedling height, RL, longest root length, SFW, seedling fresh weight, STFW, shoots fresh weigh, RFW, root fresh weight, SDW, seedling dry weight, STDW - shoots dry weight, RDW, root dry weight, RSFWR, root-to-shoot fresh weight ratio, RSDWR, root-to-shoot dry weight ratio. Small letters 'a' and 'b' means significance of difference when $P<0.05$, and capital letters ' $A$ ' and ' $B$ ' means significance of difference when $P<0.01$. Difference is significant or highly significant when the small or capital letters are different between control and treatment, and difference is not significant when the letters are same. ${ }^{n}$ The number of biological replicates for RILs and both parents were all three.

biological yield factor. The measurement value drought tolerance (DV) for each line and parents was calculated by the subordinate function value of each trait multiplied by the eigenvector of corresponding trait of the first principal component and then added.

\section{Evaluation of drought tolerance}

The DV of RIL populations and its parents ranged from 0.63 to 2.22. The DVs of Weimai 8 and Luohan 2 were 0.17 and 1.39 , respectively. The drought tolerance of 245 lines and two parents was divided into three types by $\mathrm{K}$ - means clustering: drought sensitive (DV ranging from 0.29 to 1.11), medium drought tolerance (DV ranging from 1.12 to 1.48), and high drought tolerance (DV ranging from 1.49 to 2.22). The parent Weimai 8 and 101 lines were clustered into drought-sensitive types, Luohan 2 and 102 lines were clustered into medium drought tolerance lines, and 42 other lines were clustered into highly drought-tolerance types (Table 3 ). These results validated previous information that the drought tolerance of Luohan 2 is better than that of Weimai 8 , which is in accordance with production practices. Although Weimai 8 was drought-sensitive, some superior plants with good variance were produced by the hybridization between two 
Table 2. Eigenvectors and percentages of accumulated contribution of principal components.

\begin{tabular}{lcccc}
\hline Trait & Component 1 & Component 2 & Component 3 & Component 4 \\
\hline CL & 0.065 & 0.099 & -0.016 & $-0.238^{*}$ \\
SH & 0.135 & 0.207 & -0.082 & $-0.218^{*}$ \\
RL & 0.447 & -0.096 & -0.427 & $-0.595^{*}$ \\
SFW & $0.284^{*}$ & 0.165 & -0.081 & 0.104 \\
STFW & 0.212 & $0.304^{*}$ & 0.058 & -0.030 \\
RFW & $0.396^{*}$ & -0.042 & -0.288 & 0.320 \\
SDW & $0.318^{*}$ & 0.303 & 0.257 & 0.163 \\
STDW & 0.221 & $0.443^{*}$ & 0.026 & 0.127 \\
RDW & 0.463 & -0.047 & $0.492^{*}$ & 0.029 \\
RSFWR & 0.256 & -0.417 & -0.418 & $0.460^{*}$ \\
RSDWR & 0.257 & $-0.594^{*}$ & 0.471 & -0.174 \\
Eigenvector & 6.919 & 4.875 & 1.652 & 1.167 \\
Contribution rate & 0.4080 & 0.2876 & 0.0975 & 0.0688 \\
Accumulated contribution rate & 0.4080 & 0.6956 & 0.7931 & 0.8619 \\
\hline
\end{tabular}

*, Biggest absolute value of each index in all factors.

parents. The drought tolerance of $15 \%$ of the lines in this RIL population was better than that of Luohan 2 . Selection of these lines provides valuable material for wheat drought tolerance breeding.

\section{Relativity analysis of DV and drought tolerance index of each trait}

The correlation coefficients among tolerance indexes of 11 seedling traits and DV were calculated to determine the most desirable drought-tolerant criteria (Table 4). The results indicate that significant or highly significant correlations were observed among the drought tolerance of other traits, except those between CLI and RFWI, between SFWI and RSFWRI, RSDWRI, between SDWI and RSFWRI, RSDWRI, all of which were not significant. DV had a highly significant positive correlation with CLI, SHI, RLI, SFWI, SDWI, STFWI, STDWI, RFWI, RDWI, RSFWRI, and RSDWRI. These findings indicate that DV could represent the drought tolerance information of all seedling traits so it could be used as an evaluation indicator. In addition, DV had greater correlation coefficients with RFWI, RDWI, and RLI, yielding values of $0.868,0.850$, and 0.795 , respectively. These results indicated that the response of root systems for drought tolerance was the most sensitive. Whether or not a relatively strong root system could be grown appeared to be an important condition for evaluating seedling for drought tolerance.

\section{DISCUSSION}

Analysis for drought tolerance could use either single or multiple indicators. Single-indicator analysis at the seedling stage of wheat often uses the drought tolerance index and the survival rate of seedlings after repeated droughts, among others. These kinds of indicator are simple and easily implementable; however, they reflect the responses of few traits for drought tolerance. Multipleindicator analysis could reflect the characteristics of drought tolerance with different aspects. Principal component analysis is an important method that is often been used for such determinations (Atefeh et al., 2011; Reza et al., 2011). The method discards unnecessary indicators, selects essential factors, and establishes a reasonable weighted coefficient for every trait indicator, thereby allowing the evaluation to be more scientific and reasonable. Meng (1992) pointed out that the value of the first principal component which was a comprehensive index obtained through principal component analysis and included maximum information of original indexes and not that of the comprehensive principal component, should be considered the comprehensive evaluation value. In this present paper, four components with a total contribution of $86.19 \%$ (Table 2) were selected using principal component analysis for a subordinate function of 11 seedling traits. Among these components, the contribution rate of the first principal component was $40.8 \%$. The evaluation of drought tolerance for each line and parent was carried out using the first principal component as the comprehensive evaluation value. Two hundred and ninety-one lines and two parents were classified into three types. The analysis followed the theory of statistics, excluding the interference of subjective factors; these steps ensured that the results were both objective and reliable.

$\mathrm{CL}$ is an important indicator in evaluating the drought tolerance of seedlings. Research has shown that $\mathrm{CL}$ under water stress has highly significant positive 
Table 3. Drought tolerance classification of RIL population and its parents.

\begin{tabular}{|c|c|c|c|c|c|c|c|c|c|c|c|c|c|c|c|c|c|c|c|}
\hline Type & & Num & of line & & & & & & & & & & & & & & & & \\
\hline \multirow[t]{3}{*}{ Drought } & 17 & 248 & 88 & 160 & Weimai 8 & 293 & 245 & 36 & 60 & 197 & 122 & 14 & 135 & 155 & 100 & 156 & 143 & 184 & \\
\hline & 260 & 199 & 105 & 34 & 291 & 142 & 174 & 246 & 44 & 294 & 118 & 276 & 271 & 191 & 269 & 129 & 49 & 131 & \\
\hline & & 56 & 186 & 290 & 223 & 169 & 48 & 13 & 133 & 198 & 5 & 195 & 98 & 254 & 158 & 279 & 43 & 20 & 128 \\
\hline \multirow[t]{3}{*}{ Sensitive } & & 64 & 140 & 282 & 38 & 132 & 84 & 55 & 206 & 22 & 180 & 242 & 72 & 277 & 161 & 288 & 302 & 209 & 190 \\
\hline & & 179 & 274 & 232 & 239 & 188 & 235 & 227 & 222 & 11 & 233 & 67 & 243 & 267 & 228 & 26 & 112 & 177 & 150 \\
\hline & & 4 & 28 & 256 & 9 & 175 & 68 & 101 & 151 & 90 & 30 & 47 & & & & & & & \\
\hline Mediate & 201 & 10 & 141 & 187 & 229 & 240 & 40 & 192 & 66 & 62 & 244 & 236 & 46 & 165 & 193 & 70 & 200 & 172 & 213 \\
\hline \multirow[t]{3}{*}{ Drought } & 19 & 95 & 218 & 41 & 82 & 33 & 54 & 173 & 298 & 148 & 144 & 207 & 247 & 154 & 182 & 203 & 45 & 234 & \\
\hline & & 8 & 157 & 123 & 285 & 114 & 53 & 153 & 255 & 252 & 108 & 23 & 208 & 21 & 168 & 261 & 15 & 204 & 268 \\
\hline & & 31 & 214 & 181 & 211 & 81 & 292 & 217 & 185 & 286 & 266 & 259 & 163 & 221 & 6 & 124 & 89 & 205 & 147 \\
\hline \multirow{5}{*}{ Tolerance } & & 76 & 241 & 80 & 127 & 202 & 107 & 27 & 250 & 237 & 1 & 85 & 25 & 110 & 12 & 171 & 50 & 258 & 2 \\
\hline & & 97 & 295 & 113 & 121 & 51 & 3 & 189 & 94 & 145 & 194 & 167 & 162 & 29 & 7 & 275 & 24 & 130 & 52 \\
\hline & & 152 & 170 & 164 & 87 & 272 & Luohan 2 & 86 & 238 & 120 & 117 & 74 & 230 & 115 & 219 & 283 & 176 & 109 & \\
\hline & & 99 & 65 & 196 & 138 & 257 & 91 & 125 & 300 & 106 & 96 & 32 & 92 & 220 & 284 & 216 & 253 & 37 & 79 \\
\hline & & 225 & 210 & 149 & 59 & & & & & & & & & & & & & & \\
\hline High & 146 & 137 & 78 & 215 & 61 & 212 & 75 & 183 & 136 & 287 & 301 & 77 & 73 & 251 & 297 & 262 & 299 & 104 & 18 \\
\hline Drought & & 289 & 134 & 63 & 93 & 111 & 226 & 159 & 231 & 139 & 126 & 119 & 281 & 71 & 39 & 296 & 16 & 280 & 249 \\
\hline
\end{tabular}

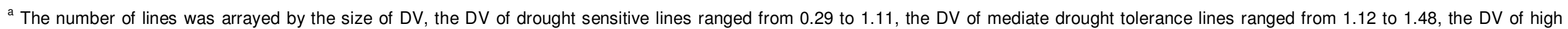
drought tolerance lines ranged from 1.49 to 2.22. Weimai 8 and Luohan 2 are two parents.

correlations with yield and that the coleoptiles of cultivars with strong drought tolerance grow quickly under osmotic stress. Thus, the method of $\mathrm{CL}$ under low water potential was brought forward for use in determining the drought tolerance for winter wheat (Wang et al., 1997; Zou et al., 2000). In this present paper, the growth speed of Luohan 2 was greater than that of Weimai 8 under osmotic stress conditions, which further validates the effect of drought on the CL of different cultivars. In addition, while the drought tolerance index of the coleoptile had highly significant positive correlations with DV, the correlation coefficient was only 0.26 , lower than those for other traits. Whether it is reasonable to use $\mathrm{CL}$ as the only indicator of drought tolerance requires further exploration.

Several studies (Siddique et al., 1989; Sharma and Lafever, 1992) have indicated that the underground part of the plant plays an important role in surviving drought stress conditions. Yang et al. (2009) found that maize varieties with stronger rooting and germination abilities have high drought tolerance. Jing et al. (1997) found that the drought tolerance of wheat seedlings has highly significant correlations with the dry weight of roots. In this present paper, the drought tolerance indexes of $R L, R F W$, and $R D W$ were found to feature highly significant positive correlations with DV, in which the highest coefficient among all of the traits was detected. This finding indicates that root traits are the most sensitive to drought and these should be the most important target traits for the identification of drought tolerance at the seedling stage, in accordance with previous studies. 
Table 4. Correlation coefficient between drought-tolerance index of each trait and DV value.

\begin{tabular}{|c|c|c|c|c|c|c|c|c|c|c|c|c|}
\hline & $X_{C L}$ & $\mathrm{X}_{\mathrm{SH}}$ & $X_{R L}$ & $X_{\mathrm{SFW}}$ & $X_{\text {STFW }}$ & $X_{\text {RFW }}$ & $X_{\text {SDW }}$ & $\mathbf{X}_{\text {STDW }}$ & $X_{\text {RDW }}$ & $\mathrm{X}_{\mathrm{RSFW}}$ & $\mathrm{X}_{\mathrm{RSDW}}$ & DV \\
\hline$X_{C L}$ & 1 & & & & & & & & & & & \\
\hline XSH & $0.605^{* *}$ & 1 & & & & & & & & & & \\
\hline XRL & $0.226^{* *}$ & $0.377^{\star *}$ & 1 & & & & & & & & & \\
\hline XSFW & $0.172^{* *}$ & $0.518^{* *}$ & $0.485^{\star *}$ & 1 & & & & & & & & \\
\hline XSTFW & $0.212^{* *}$ & $0.561^{* *}$ & $0.262^{* *}$ & $0.900^{* *}$ & 1 & & & & & & & \\
\hline XRFW & 0.080 & $0.310^{* *}$ & $0.628^{* *}$ & $0.807^{* *}$ & $0.473^{* *}$ & 1 & & & & & & \\
\hline XSDW & $0.288^{* *}$ & $0.502^{* *}$ & $0.330^{* *}$ & $0.570^{* *}$ & $0.551^{* *}$ & $0.427^{* *}$ & 1 & & & & & \\
\hline XSTDW & $0.350^{* *}$ & $0.620^{* *}$ & $0.220^{* *}$ & $0.547^{* *}$ & $0.611^{* *}$ & $0.301^{* *}$ & $0.854^{* *}$ & 1 & & & & \\
\hline XRDW & $0.177^{* *}$ & $0.268^{* *}$ & $0.526^{* *}$ & $0.518^{* *}$ & $0.351^{* *}$ & $0.586^{* *}$ & $0.734^{* *}$ & $0.393^{* *}$ & 1 & & & \\
\hline XRSFWR & $-0.128^{*}$ & $-0.193^{* *}$ & $0.447^{* *}$ & 0.104 & $-0.309^{* *}$ & $0.632^{* *}$ & -0.037 & $-0.234^{* *}$ & $0.327^{* *}$ & 1 & & \\
\hline XRSDWR & $-0.117^{*}$ & $-0.268^{* *}$ & $0.332^{* *}$ & 0.028 & $-0.189^{\star *}$ & $0.307^{* *}$ & $-0.124^{*}$ & $-0.477^{\star *}$ & $0.570^{\star *}$ & $0.528^{* *}$ & 1 & \\
\hline DV & $0.260^{* *}$ & $0.461^{* *}$ & $0.795^{\star *}$ & $0.766^{\star *}$ & $0.513^{\star *}$ & $0.868^{* *}$ & $0.660^{* *}$ & $0.460^{\star *}$ & $0.850^{\star *}$ & $0.494^{* *}$ & $0.417^{\star *}$ & 1 \\
\hline
\end{tabular}

$\mathrm{X}$, the drought-resistance index of trait, subscript is the abbreviation of seedling trait; ${ }^{* \star}$, correlation is significant when $p<0.01$ level. ${ }^{*}$, correlation is significant when $p<0.05$ level.

\section{Conclusion}

In summary, 245 lines of RIL population and two parents were divided into three clusters using principal component analysis and K-means clustering. Root traits were the most susceptible to drought, so whether or not a relatively strong root system could be grown was an important condition in evaluating the drought tolerance of wheat at the seedling stage.

\section{ACKNOWLEDGMENTS}

This research was supported by the National Basic Research Program of China (863 Program, 2011AA100103) and Creation and Utilization of Agriculture-Biology Resource of Shandong Province, China.

Abbreviations: RIL, Recombination inbred line; CL, coleoptile length; $\mathbf{S H}$, seedling height; RL, longest root length; SFW, seedling fresh weight; STFW, shoots fresh weight; RFW, root fresh weight; SDW, seedling dry weight; STDW, shoots dry weight; RDW, root dry weight; RSFWR, root-to-shoot fresh weight ratio; RSDWR, rootto-shoot dry weight ratio; DV, measurement value of drought-tolerance; CLI, tolerance index of CL; SHI, tolerance index of SH; RLI, tolerance index of RL; SFWI, tolerance index of SFW; STFWI, tolerance index of STFW; RFWI, tolerance index of RFW; SDWI, tolerance index of SDW; STDWI, tolerance index of STDW; RDWI, tolerance index of RDW; RSFWRI, tolerance index of RSFWR; RSDWRI, tolerance index of RSDWR.

\section{REFERENCES}

Almansouri M, Kinet JM, Lutts S (2001). Effect of salt and osmotic stresses on germination in durum wheat (Triticumdurum Desf). Plant Soil. 231:243-254.
Atefeh N, Alireza E, Jaime A, Teixeirada S, Reza M (2011). Assessment of yield, yield-related traits and drought tolerance of durum wheat genotypes (Triticum turjidum var. durum Desf.). Aust. J. Crop Sci. 5:816.

Blum A (1996). Crop responses to drought and the interpretation of adaptation. Plant Growth Regul. 20:135-148.

Bouslama M, Schapaugh WT (1984). Stress tolerance in soybeans. I. Evaluation of three screening techniques for heat and drought tolerance. Crop Sci. 24:933-937.

Dhanda SS, Sethi GS, Behl RK (2004). Indices of drought tolerance in wheat genotypes at early stages of plant growth. Agron. Crop Sci. 190:6-12.

He XY, Wen RL, Wu CR, Zhou JG (2008). Analysis of maize drought resistance at seeding stage by fuzzy subordination method. Southwest China J. Agric. Sci. 21:52-56.

Jing RL, Hu RH, Zhu ZH (1997). A study on heritabilities of seedling morphological traits and drought resistance in winter wheat cultivars of different genotype. Acta. Bot. Boreali-Occid. Sin. 17:152-157.

Meng SW (1992). Two problems of more comprehensive evaluation indicators in principal components analysis. Stat. Res. 4:67-68.

Michel BE, Kaufmann MR (1973). The osmotic potential of polyethylene glycol 6000. Plant Physiol. 51:914-916.

Mujtaba SM, Khanzada B, Ali M, Naqvi MH, Mughal S, Alam SM, Shereen A (2005). The effect of polyethylene glycol on seed germination of wheat (Triticum aestivum L.) genotypes lines. Wheat Inform. Serv. 99:58-60.

Reza GA, Hamdollah KA, Mehrdad Y, Golamreza A, Leila GA. Taregh G (2011). Evaluation of drought tolerance indices and grain yield in wheat genotypes using principle components analysis. Middle-East $\mathrm{J}$. Sci.Res. 8:880-884.

Siddique KHM, Belford RK, Perry MW, Tennant D (1989). Growth, development and light interception of old and modern wheat cultivars in a Mediterranean-type environment. Aust. J. Agric. Res. 40:473487.

Sharma RC, Lafever NN (1992). Variability for root traits and their genetic contribution in spring wheat. Euphytica 59:1-8.

Wang W, Zou Q, Yang XH, Peng T, Li Y (1997). Studies on the relativity among coleoptile length, osmotic adjustment and yield in wheat under water stress. Chin. Bull. Bot. 14:55-59.

Yang GH, Wang WH, Song HX, Li YQ, Qi H, Wang TY, Zhao JR. (2009). Analysis of drought resistance of maize hybrids in rain-field farming. Crops 5:78-81.

Zou Q, Wang W, Yang XH (2000). New method of identification of drought resistance in winter wheat-the method of coleoptile length under water stresses. Chin. Agric. Sci. Bull. 16:23-25. 